Relations industrielles

Industrial Relations

\title{
Lexique des sciences sociales, par Madeleine Grawitz, Paris, Dalloz, 1981, 376 pp.
}

\section{Dimitri Weiss}

Volume 36, numéro 4, 1981

URI : https://id.erudit.org/iderudit/029215ar

DOI : https://doi.org/10.7202/029215ar

Aller au sommaire du numéro

Éditeur(s)

Département des relations industrielles de l'Université Laval

ISSN

0034-379X (imprimé)

1703-8138 (numérique)

Découvrir la revue

Citer ce compte rendu

Weiss, D. (1981). Compte rendu de [Lexique des sciences sociales, par Madeleine Grawitz, Paris, Dalloz, 1981, 376 pp.] Relations industrielles / Industrial

Relations, 36(4), 945-946. https://doi.org/10.7202/029215ar

Tous droits réservés @ C Département des relations industrielles de l'Université Laval, 1981
Ce document est protégé par la loi sur le droit d'auteur. L'utilisation des services d'Érudit (y compris la reproduction) est assujettie à sa politique d'utilisation que vous pouvez consulter en ligne.

https://apropos.erudit.org/fr/usagers/politique-dutilisation/ 
définies. Ces concepts sont sujets à de nombreuses interprétations; il est relativement facile de bâtir autour d'eux des explications théoriques; il est cependant beaucoup plus difficile d'être précis et de trouver des façons adéquates de mesurer et de vérifier ses affirmations dans la réalité. Pour faire oeuvre utile dans ce domaine, un auteur doit se méfier des affirmations faciles et gratuites; il doit donc être à la fois systématique et rigoureux. Et c'est en faisant preuve d'une grande rigueur que B.J. Briddle démontre dans son volume la pertinence ainsi que l'utilité des concepts associés à la notion de rôle.

En ce qui a trait à l'organisation du contenu, l'auteur utilise une approche allant du plus simple au plus complexe. Dans les premiers chapitres, en effet, tout l'aspect cognitif ou intrapersonnel de la théorie des rôles est volontairement mis de côté pour ne considérer que les éléments ou comportements directement observables. C'est ainsi que la notion de rôle y est définie comme étant... "those behaviors characteristic of one or more persons in a context". Ce n'est qu'à partir du chapitre IV que l'auteur laisse progressivement entrer des concepts plus complexes comme ceux de position et d'identité. Les chapitres $\mathrm{V}$ et VI sont consacrés aux phénomènes cognitifs que sont les attentes et aux nombreux concepts reliés à cette façon de définir la notion de rôle. (L'auteur introduit ici le concept de "expected role"). Le chapitre VII concerne l'application des concepts de la théorie des rôles à l'analyse d'un système social. Le chapitre VIII concerne les phénomènes de socialisation, d'ajustement, d'adaptation et d'influence entre la personne et le contexte social dans lequel elle évolue. Le chapitre IX, enfin, constitue la conclusion: l'auteur y présente, entre autres choses, une liste des tâches à effectuer pour continuer de faire évoluer la théorie des rôles.

Le présent volume est d'une lecture relativement facile et agréable. À de nombreux endroits, l'auteur fait preuve d'une culture large et éclairée. De plus, le vocabulaire utilisé est clair et dépourvu du jargon que d'autres auteurs dans ce domaine ont si souvent tendance à utiliser.
Même si la revue de la documentation spécialisée est surtout axée sur la sociologie et que certaines revues en administration et en sciences du comportement semblent avoir été négligées (en ce sens que de nombreux articles reliés à la théorie des rôles ne sont pas répertoriés), Role Theory apparaît comme une contribution significative à une meilleure compréhension de ce domaine de recherche. Les chercheurs et étudiants en sociologie, anthropologie, psychologie sociale, sciences du comportement, counseling, comportement organisationnel, etc. trouveront profit à en faire la lecture.

André PETIT

Université de Sherbrooke

Lexique des sciences sociales, par Madeieine Grawitz, Paris, Dalloz, 1981, 376 pp.

Ce lexique - c'est-à-dire, selon la définition même de l'auteur (page 230), «recueil comprenant une liste de termes utilisés pour décrire le contenu d'un champ sémantique quelconque» - était attendu, et il est le bienvenu.

Nous concerne-t-il dans notre multi (ou inter) discipline? Oui, sans le moindre doute.

D'abord, le terme même de relations industrielles y figure-t-il? Oui, de façon lapidaire, sous le vocable relation, placé entre relations humaines et relations internationales, et défini - à l'ancienne - comme l' «étude des relations entre employeurs et employés, des facteurs d'influence capables de créer un climat de collaboration».

Mais lorsque l'on regarde le mot industrialisme, ce n'est pas dans le sens utilisé par Dunlop, Harbison, Kerr et Myers - et par beaucoup d'autres, d'ailleurs - mais dans la signification que lui a donnée Saint-Simon en 1915 qu'il nous est rendu, qui qualifiait ainsi sa doctrine opposant non pas les patrons aux ouvriers, mais les producteurs (ouvriers, chefs d'industrie, paysans, intellectuels, etc.) aux notables, propriétaires fonciers.

On trouve certes, au fil des pages, décrits avec des bonheurs divers, absentéisme, chô- 
mage, cogestion, démocratie (industrielle: "concerne les projets et tentatives de réformes du capitalisme et du pouvoir dans l'entreprise»), emploi, grève (dans le sens large, avec toute une énumération de formes), groupe (sur plusieurs pages), lock-out ("mettre dehors. Fermeture d'une usine ou d'un atelier", sans aucune référence à la grève), organisation, ouvrier et ouvriérisme, participation, patron, politique (avec un utile développement sur ce qui sépare le politique de la politique), psychosociologie, qualification, questionnaire, salarié, stress, syndicalisme, synergie (mais pas dans le sens donné par Ansoff) et travail.

Ce n'est bien entendu pas pour ces termes, qui nous concernent directement, et dont la description me semble par endroits non satisfaisante, que je recommande l'utilisation de ce lexique aux observateurs des relations industrielles. Le dictionnaire («suite de définitions, intermédiaire entre lexique qui renvoie à des termes et encyclopédie à des connaissances») canadien des relations du travail, de Gérard Dion, reste à cet égard, et pour longtemps, un instrument insubstituable. Mais pour beaucoup d'autres, là ou l'auteur est dans son élément, où elle montre la grande maîtrise qui a fait sa réputation, et qu'elle met à la disposition du grand nombre.

Car, ainsi qu'elle l'écrit dans l'avantpropos, "ce lexique s'adresse à un public très large de lecteurs, susceptibles de rencontrer des termes de sciences sociales sans avoir étudié celles-ci, mais également aux spécialistes d'un seul domaine». Nous sommes là bien concernés - le département de relations industrielles de Laval ne fait-il pas partie d'une faculté des sciences sociales? -, et d'autant plus que notre domaine, pluridisciplinaire, c'est-à-dire qui «implique seulement la collaboration de plusieurs disciplines», selon certaines opinions ou (comme je le crois) interdisciplinaire, qui rassemble «ce qui est commun à plusieurs disciplines, qui crée un lien entre elles» (page 214), n'est, par définition, pas circonscrit entre des frontières rigides.

À quoi s'ajoute le besoin de savoir de chacun d'entre nous. En ce sens, le double objectif poursuivi par Madeleine Grawitz me semble atteint: transmettre des connaissances, mêmes limitées, et contribuer à la formation de l'esprit.

J'ajoute que ce volume, paru en format de poche, est aisément manipulable.

Dimitri WEISS

Université du Maine

et Institut d'Administration des

Entreprises (Université de Paris I

Panthéon-Sorbonne)

Organisations sociales et comportements, par Jean M. Guiot, Montréal, Les éditions Agence d'Arc Inc. et les éditions Hommes et Techniques, Paris, 1980, 213 pp.

Pour donner un compte rendu assez complet des différents apports de la sociologie, de la psychosociologie et de la psychologie à l'étude des organisations, il aurait fallu rédiger une encyclopédie comprenant plusieurs tomes et des milliers de pages. Dans cet ouvrage de 200 quelques pages, Jean M. Guiot a réussi à relever un défi en présentant d'une façon concise et précise les contributions les plus connues à l'étude des objectifs, des structures et du fonctionnement des organisations. Arnold S. Tonnenbaum en rédigeant la préface annonce que l'auteur a voulu expliquer "certaines contradictions ou «irrationalités» qui contribuent au décalage entre le formel et le vécu dans le fonctionnement organisationnel». Il faut s'attendre là à des explications, non pas tirées de recherches sur le terrain, mais bien de modèles existants dont la plupart sont descriptifs et quelquesuns normatifs. Comme il le souligne luimême, l'auteur s'inspire du modèle de Leavitt qui retient cinq grandes catégories de variables interdépendantes: les objectifs, les structures, la technologie, l'environnement et la composition sociale des membres de l'organisation. Ces grandes variables font l'objet des six premiers chapitres; les autres traitent des relations de pouvoir, des motivations, des perceptions, du leadership, de la satisfaction au travail et du développement organisationnel. Bien entendu, ce sont là des thèmes déjà traités dans une foule d'ouvrages d'origine américaine surtout. Cependant, ce qui est 